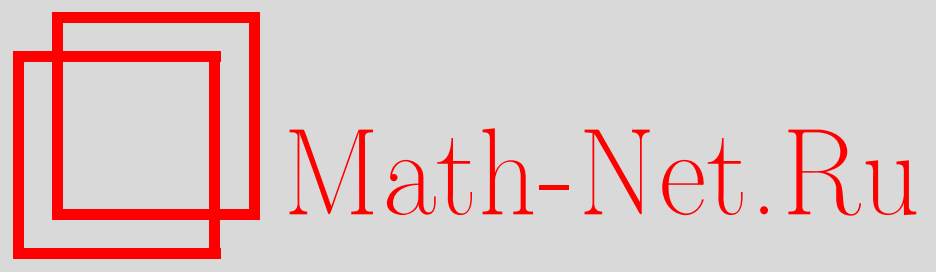

Е. В. Троицкий, М. Франк, Числа Лефшеца и геометрия операторов в $W^{*}$-модулях, Функи. анализ и его прил., 1996, том 30, выпуск 4, 45-57

DOI: https://doi.org/10.4213/faa549

Использование Общероссийского математического портала MathNet.Ru подразумевает, что вы прочитали и согласны с пользовательским соглашением

http://www . mathnet.ru/rus/agreement

Параметры загрузки:

IP : 52.6 .47 .48

26 апреля 2023 г., 12:54:22

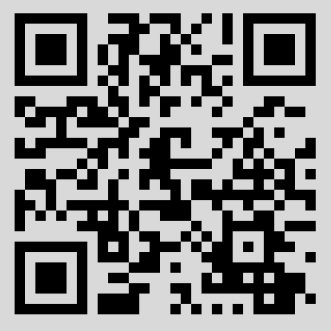




\title{
Числа Лефшеца и геометрия операторов в $\mathrm{W}^{*}$-модулях ${ }^{\star}$
}

\author{
(c) 1996. Е. В. ТРоицкий, М. ФРАнк
}

\section{§1. Введение}

Основной целью этой работы является обобщение результатов $[18,19]$ в следующем направлении. Для того чтобы определить $K_{0}(A) \otimes \mathbb{C}$-значные числа Лефшеца (первого типа) эндоморфизма $V \mathrm{C}^{*}$-эллиптического комплекса, обычно предполагают, что $V=T_{g}$ для некоторого представления $T_{g}$ компактной группы $G$ на $\mathrm{C}^{*}$-эллиптическом комплексе. Мы постараемся в настоящей работе отказаться от этого ограничения. За это нам придется заплатить дважды:

(1) мы вынуждены определять числа Лефшеца со значениями в некоторой группе, большей, чем $K_{0}(A) \otimes \mathbb{C}$;

(2) мы должны работать с $\mathrm{W}^{*}$-алгебрами вместо общих унитальных $\mathrm{C}^{*}$-алгебр.

При получении основных результатов мы доказали ряд вспомогательных утверждений о гильбертовых $\mathrm{W}^{*}$ - и $\mathrm{C}^{*}$-модулях и ограниченных модульных операторах в них, которые могут представлять самостоятельный интерес.

Настоящая работа устроена следующим образом. В $\S 2$ мы доказываем необходимые факты о гильбертовых $\mathrm{W}^{*}$-модулях и их ограниченных модульных отображениях, усиливающие результаты В. Л. Пашке [14], Ж.-Ф. Хаве [5] и М. Франка [3]. В $\S 3$ мы определяем числа Лефшеца двух типов и устанавливаем их основные свойства. В $\S 4$ обсуждается $\mathrm{C}^{*}$-случай и препятствия к улучшению результатов $\S 3$.

Нашими стандартными источниками по теории гильбертовых $\mathrm{C}^{*}$-модулей будут служить статьи $[2,3,9-11,14,15]$ и книга Лэнса [8]. Основой для топологических построений являются работы $[11-13,17-19]$. Мы надеемся продолжить настоящее исследование.

Авторы признательны Немецкой службе академических обменов (DAAD) за предоставленную возможность совместной работы в Лейпцигском университете в соответствии с локальным проектом «Некоммутативная геометрия». Авторы благодарят Й. Кунтца, В. М. Мануйлова, А. С. Мищенко и Р. Неста за полезные обсуждения.

\section{§2. Гильбертовы $\mathrm{W}^{*}$-модули и их морфизмы}

Мы хотим установить некоторые весьма полезные свойства гильбертовых $\mathrm{W}^{*}$-модулей, которые зачастую не имеют места в общем $\mathrm{C}^{*}$-случае. Внимание специалистов было привлечено к этому специальному классу гильбертовых

* Исследование первого автора было частично поддержано Российским фондом фундаментальных исследований (грант №94-01-00108-а) и грантом No. MGM300 Международного научного фонда и Российского правительства. 
$\mathrm{C}^{*}$-модулей В. Л. Пашке в его классической работе [14], после чего этот объект стал использоваться во многих ситуациях. Факты, о которых пойдет речь ниже, могут быть установлены и для монотонно полных $\mathrm{C}^{*}$-алгебр, хотя это и требует большой технической работы (ср. [4]), но, вообще говоря, не для более широкого класса $\mathrm{C}^{*}$-алгебр. Однако, поскольку мы собираемся разобраться в структуре обших гильбертовых $\mathrm{C}^{*}$-модулей и $\mathrm{C}^{*}$-двойственных к ним, то лучше заниматься общим $\mathrm{W}^{*}$-случаем и обойти эти сложности.

На протяжении этого параграфа мы будем обозначать через $A$ произвольную $\mathrm{W}^{*}$-алгебру, через $\{\mathscr{M},\langle\cdot, \cdot\rangle\}$ и $\mathscr{N}, \mathscr{P}, \mathscr{Q}$ гильбертовы $A$-модули и через $\widehat{\oplus}$ прямую ортогональную сумму двух гильбертовых $A$-модулей, в то время как $\oplus$ обозначает прямую топологическую сумму гильбертовых $A$-подмодулей некоторого гильбертова $A$-модуля без требования ортогональности двух компонент. Морфизмы модулей будут обозначаться греческими буквами. Мы начнем со свойства, обобщающего свойство двойного аннулятора произвольных множеств в $\mathrm{W}^{*}$-алгебрах.

ЛЕмма 1. Для каждого подмножества $\mathscr{S} \subseteq \mathscr{M}$ биортогональное множество $\mathscr{S}^{\perp \perp} \subseteq \mathscr{M}$ является гильбертовьм А-подмодулем и прямым слагаемьлм А-модуля $\mathscr{M}$, равно как и его ортогональное дополнение $\mathscr{S}^{\perp}$.

ДоказАтельство. Тот факт, что $\mathscr{S}^{\perp \perp} \subseteq \mathscr{M}$ является $A$-подмодулем, очевиден по определению ортогональных дополнений. Поскольку $A$-сопряженный банахов $A$-модуль $\mathscr{M}^{\prime}$ к $\mathscr{M}$ является автодуальным гильбертовым $A$-модулем по [14, Theorem 3.2], содержащим $\mathscr{M}$ при стандартном вложении, то можно рассмотреть гильбертов $A$-подмодуль $\mathscr{N}=\mathscr{S}^{\perp \perp} \widehat{\oplus}\left(\mathscr{S}^{\perp \perp}\right)_{\mathscr{M}^{\prime}}^{\perp} A$-модуля $\mathscr{M}^{\prime}$. Второе слагаемое является по построению ортогональным дополнением к $\mathscr{S}^{\perp \perp}$ в $\mathscr{M}^{\prime}$ и, следовательно, автодуальным гильбертовым $A$-подмодулем и прямым слагаемым в $\mathscr{N}$ по [3, теоремы 3.2 и 2.8]. Значит, образ канонического вложения $A$-модуля $\mathscr{S}^{\perp \perp}$ в $\mathscr{N}$ является прямым слагаемым в $\mathscr{N}$, а в силу включения подмодулей $\mathscr{S}^{\perp \perp} \subseteq \mathscr{M} \hookrightarrow \mathscr{N}$ - прямым слагаемым и в $\mathscr{M}$.

Пример 1 ниже показывает, что ситуация, отличная от описанной в лемме 1 , может возникнуть, например, для гильбертовых $\mathrm{C}^{*}$-модулей над $\mathrm{C}^{*}$-алгеброй $A=C([0,1])$.

ЛЕмма 2. Пусть $\phi-$ ограниченный модульный морфизм $A$-модуля $\mathscr{M}$. Тогда лдро $\operatorname{Ker}(\phi)$ отображения $\phi$ являетсл прлмьм слагаемым в $\mathscr{M} и$ удовлетворяет соотношению $\operatorname{Ker}(\phi)=\operatorname{Ker}(\phi)^{\perp \perp}$.

ДокаЗАтЕльство. По [14, Proposition 3.6] каждое ограниченное модульное отображение $\phi$ модуля $\mathscr{M}$ продолжается до ограниченного модульного отображения его $A$-сопряженного гильбертова $A$-модуля $\mathscr{M}^{\prime}$. Ядро продолженного оператора является прямым слагаемым модуля $\mathscr{M}^{\prime}$ в силу полноты его единичного шара относительно $\tau_{2}$-сходимости, порожденной там функционалами $\left\{f(\langle\cdot, y\rangle): f \in A_{*, 1}, y \in \mathscr{M}^{\prime}\right\}$ (ср. [3, Theorem 3.2]). Следовательно, ядро морфизма $\phi$ в $\mathscr{M}$ должно совпадать с его биортогональным дополнением в $\mathscr{M}$, и по лемме 1 оно является прямым слагаемым.

ПримеР 1. Заметим, что, вообще говоря, ядро ограниченного $A$-линейного оператора в гильбертовом $A$-модуле над произвольной $\mathrm{C}^{*}$-алгеброй $A$ не является прямым слагаемым. Например, рассмотрим $\mathrm{C}^{*}$-алгебру $A=C([0,1])$ всех 
непрерывных функций на отрезке $[0,1]$ как гильбертов $A$-модуль над ней самой, снабженный стандартным скалярным произведением $\langle a, b\rangle_{A}=a b^{*}$. Определим отображение $\phi_{g}$ формулой $\phi_{g}(f)=g \cdot f$ для фиксированной функции

$$
g(x)= \begin{cases}-2 x+1, & x \leqslant 1 / 2, \\ 0, & x \geqslant 1 / 2,\end{cases}
$$

и всякого $f \in A$. Тогда $\operatorname{Ker}\left(\phi_{g}\right)$ совпадает с гильбертовым $A$-подмодулем и (левым) идеалом $\{f \in A: f(x)=0$ для $x \in[0,1 / 2]\}$, не является прямым слагаемым в $A$, однако совпадает с биортогональным дополнением к себе в $A$.

СЛЕДСТВИЕ 1. Пусть $\phi: \mathscr{M} \rightarrow \mathscr{N}$ - ограниченное A-линейное отображение. Тогда ядро $\operatorname{Ker}(\phi)$ отображения $\phi$ является прямьм слагаемьм в $\mathscr{M}$ и удовлетворяет соотношению $\operatorname{Ker}(\phi)=\operatorname{Ker}(\phi)^{\perp \perp}$.

ДокАЗАтельство. Рассмотрим гильбертов $A$-модуль $\mathscr{K}$, полученный как прямая сумма $\mathscr{K}=\mathscr{M} \oplus \mathscr{N}$ с $A$-значным скалярным произведением $\langle\cdot, \cdot\rangle \mathscr{M}+$ $\langle\cdot, \cdot\rangle \mathscr{N}$. Отображение $\phi$ может быть отождествлено с ограниченным $A$-линейным отображением $\phi^{\prime}$ в $\mathscr{K}$, действуюшим на прямом слагаемом $\mathscr{M}$ как $\phi$, а на прямом слагаемом $\mathscr{N}$ как нулевой оператор. Поскольку ядро отображения $\phi^{\prime}$ является по лемме 2 прямым слагаемым модуля $\mathscr{K}$, содержащим $\mathscr{N}$, то его ортогональное дополнение будет прямым слагаемым в $\mathscr{M}$.

Теперь мы в состоянии дать описание внутренней структуры произвольного гильбертова $\mathrm{W}^{*}$-модуля, обобщающее аналогичное утверждение для автодуальных гильбертовых $W^{*}$-модулей, принадлежащее В. Л. Пашке [14, Theorem 3.12].

ПрЕДЛОЖЕНИЕ 1. Каждый левьй гильбертов А-модуль $\mathscr{M}$ является замькканием прямой ортогональной суммь семейства $\left\{D_{\alpha}: \alpha \in I\right\}$ замкнутых по норме левых идеалов $D_{\alpha} \subseteq A$, где замыкание этой прямой суммь определяется заданным на $\mathscr{M}$ A-значным скалярным произведением $\langle\cdot, \cdot\rangle$ $u$ где А-значнье скалярнье произведения на идеалах - это стандартнье A-значные скалярные произведения на $A$. Более того, для любого ограниченного А-линейного отображения $r: \mathscr{M} \rightarrow A$ имеется сеть $\left\{x_{\beta}: \beta \in J\right\}$ элементов из $\mathscr{M}$, для которой предел по \|\|$_{A}$

$$
\lim _{\beta \in J}\left\langle y, x_{\beta}\right\rangle
$$

существует для всякого у $\in \mathscr{M}$ и равен $r(y)$.

ДокАЗАТЕЛЬСтво. Зафиксируем произвольное ограниченное $A$-линейное отображение $r: \mathscr{M} \rightarrow A$. Его ядро является прямым слагаемым в $\mathscr{M}$ по следствию 1. Рассмотрим его ортогональное дополнение. Поскольку $r$ может быть продолжено до ограниченного $A$-линейного отображения $r(\cdot)=\left\langle\cdot, x_{r}\right\rangle$ в $A$-сопряженном к модулю $\mathscr{M}$ (автодуальном) гильбертовом $A$-модуле $\mathscr{M}^{\prime}$ $\left(x_{r} \in \operatorname{Ker}(r)^{\perp} \subseteq \mathscr{M}^{\prime}\right)$ и поскольку ортогональное дополнение ядра отображения $r$ в $\mathscr{M}^{\prime}$ является прямым слагаемым, изоморфным $\{A p,\langle\cdot, \cdot\rangle\}$ для некоторого проектора $p \in A$, по структурной теореме [14, Theorem 3.12] для автодуальных гильбертовых $W^{*}$-модулей ортогональное дополнение ядра $r$ по отношению к $\mathscr{M}$ изоморфно гильбертову $A$-модулю $\left\{I,\langle\cdot, \cdot\rangle_{A}\right\}$ для некоторого замкнутого по норме левого идеала $I \subseteq A p$ в $A$, где левое строгое замыкание левого 
идеала $I$ является ${ }^{*}$-замкнутым идеалом $A p$ в $A$. Теперь $r$ может быть отождествлено с элементом $x_{r} \in A p$, a $x_{r} \in A p$ является строгим левым пределом сети $\left\{x_{\beta}: \beta \in J\right\}$ элементов из $I \cap \mathscr{M}($ ср. $[16, \S 3.12])$.

Наконец, по трансфинитной индукции можно разложить $\mathscr{M}$ в прямую сумму попарно ортогональных прямых слагаемых вида $\operatorname{Ker}(r)^{\perp}$ для ограниченных $A$-линейных функционалов $r$ на $\mathscr{M}$, где $\operatorname{Ker}(r)^{\perp}$ всегда изоморфно левому замкнутому по норме идеалу $I$ в $A$ со стандартным $A$-значным скалярным произведением на нем.

Мы переходим к исследованию образов ограниченных модульных отображений гильбертовых $\mathrm{W}^{*}$-модулей. Как показывает следующий пример, здесь возникает много неприятностей, однако можно показать, что вложения автодуальных гильбертовых $\mathrm{W}^{*}$-модулей в гильбертовы $\mathrm{W}^{*}$-модули являются отображениями на прямые слагаемые (в отличие от ситуации с общими гильбертовыми $\mathrm{C}^{*}$-модулями).

ПримеР 2. Пусть $A-$ множество всех ограниченных линейных операторов $B(H)$ в сепарабельном гильбертовом пространстве $H$ с базисом $\left\{e_{i}: i \in \mathbb{N}\right\}$. Обозначим через $k$ оператор $k\left(e_{i}\right)=\lambda_{i} e_{i}$ для последовательности $\left\{\lambda_{i}: i \in \mathbb{N}\right\} \in$ $c_{0}(\mathbb{R})$. Тогда отображение $\phi_{k}: A \rightarrow A, \phi_{k}: a \rightarrow a \cdot k$, является ограниченным $A$-линейным отображением левого проективного гильбертова $A$-модуля $A$. Но его образ не является прямым слагаемым этого $A$-модуля и даже не гильбертов, поскольку прямые слагаемые в $A$ имеют вид $A p$ для некоторого проектора $p$ модуля $A$, a $1_{A} \cdot k$ равнялось бы $p$. Образ отображения $\phi_{k}$ является подмножеством множества всех компактных операторов в $H$. Заметим, что $\phi_{k}$ не инъективно.

Следующее предложение было доказано Х. Лином [10, Theorem 2.2] для произвольных $\mathrm{C}^{*}$-алгебр $A$, счетно порожденных гильбертовых $A$-модулей $\mathscr{M}, \mathscr{N}$ (без требования автодуальности) и инъективного ограниченного морфизма модулей $\alpha: \mathscr{M} \rightarrow \mathscr{N}$ с плотным по норме образом. Мы представляем другой вариант рассуждения для аналогичной ситуации в $\mathrm{W}^{*}$-случае.

ПрЕДЛОЖЕНИЕ 2. Пусть $\mathscr{M}$ автодуален, $a\{\mathscr{N},\langle\cdot, \cdot\rangle\}$ произволен. Предположим, что имеется инъективное ограниченное отображение модулей $\alpha: \mathscr{M} \rightarrow \mathscr{N}$, причем образ удовлетворяет соотношению $\alpha(\mathscr{M})^{\perp \perp}=\mathscr{N} \cdot$ Тогда оператор $\alpha\left(\alpha^{*} \alpha\right)^{-1 / 2}$ является ограниченным модульным изоморфизмом модулей $\mathscr{M}$ и $\mathscr{N} . B$ частности, они изоморфны как гильбертовы $A$-модули.

ДокАЗАТЕльСтво. В силу автодуальности модуля $\mathscr{M}$ (ср. [14, Proposition 3.4]) отображение $\alpha$ допускает ограниченный сопряженный модульный морфизм $\alpha^{*}: \mathscr{N} \rightarrow \mathscr{M}$. Поскольку $\alpha^{*} \alpha$ является положительным элементом $\mathrm{C}^{*}$-алгебры $\operatorname{End}_{A}(\mathscr{M})$ всех ограниченных модульных отображений гильбертова $A$-модуля $\mathscr{M}$, допускающих сопряженное, то его квадратный корень $\left(\alpha^{*} \alpha\right)^{1 / 2}$ корректно определяется пределом по $\|\cdot\|$ :

$$
\left(\alpha^{*} \alpha\right)^{1 / 2}=\lim _{n \rightarrow \infty}\left\|\left(\alpha^{*} \alpha\right)\right\|^{1 / 2}\left(\operatorname{id}_{\mathscr{M}}-\sum_{k=1}^{n} \lambda_{k}\left(\operatorname{id}_{\mathscr{M}}-\frac{\left(\alpha^{*} \alpha\right)}{\left\|\left(\alpha^{*} \alpha\right)\right\|}\right)^{k}\right)
$$

с коэффициентами $\left\{\lambda_{k}\right\}$, взятыми из разложения в ряд Тейлора в нуле комплекснозначной функции $f(x)=\sqrt{1-x}$ на интервале $[0,1]$. Более того, поскольку

$$
\left\langle\left(\alpha^{*} \alpha\right)^{1 / 2}(x),\left(\alpha^{*} \alpha\right)^{1 / 2}(x)\right\rangle=\langle\alpha(x), \alpha(x)\rangle
$$


и поскольку $\alpha$ инъективно, отображение $\left(\alpha^{*} \alpha\right)^{1 / 2}$ имеет тривиальное ядро. $\mathrm{C}$ другой стороны, достаточно лишь заметить, что образ отображения $\left(\alpha^{*} \alpha\right)^{1 / 2}$ является $\tau_{1}$-плотным в $\mathscr{M}($ ср. [3]). В самом деле, для каждого $A$-линейного ограниченного функционала $r(\cdot)=\langle\cdot, y\rangle$ на автодуальном гильбертовом $A$-модуле $\mathscr{M}$, отображающего образ отображения $\left(\alpha^{*} \alpha\right)^{1 / 2}$ в нуль,

$$
0=\left\langle\left(\alpha^{*} \alpha\right)^{1 / 2}(x), y\right\rangle=\left\langle x,\left(\alpha^{*} \alpha\right)^{1 / 2}(y)\right\rangle
$$

для всякого $x \in \mathscr{M}$. Следовательно, $y=0$, поскольку $\left(\alpha^{*} \alpha\right)^{1 / 2}$ инъективно, а $x \in \mathscr{M}$ было выбрано произвольно.

Теперь рассмотрим отображение $\alpha\left(\alpha^{*} \alpha\right)^{-1 / 2}$, определенное на $\mathscr{M}$. Поскольку $\left(\alpha^{*} \alpha\right)^{1 / 2}$ имеет как $\tau_{1}$-плотный образ, так и тривиальное ядро по предположению о $\alpha$, то обратный к нему неограниченный модульный оператор $\left(\alpha^{*} \alpha\right)^{-1 / 2}$ является $\tau_{1}$-плотно определенным. Получаем

$$
\left\langle\alpha\left(\alpha^{*} \alpha\right)^{-1 / 2}(x), \alpha\left(\alpha^{*} \alpha\right)^{-1 / 2}(y)\right\rangle=\langle x, y\rangle
$$

для всех $x, y$ из $\left(\tau_{1}\right.$-плотной) области определения отображения $\left(\alpha^{*} \alpha\right)^{-1 / 2}$. Следовательно, оператор $\alpha\left(\alpha^{*} \alpha\right)^{-1 / 2}$ продолжается до ограниченного изометрического модульного оператора на $\mathscr{M}$ по $\tau_{1}$-непрерывности. Его образ является $\tau_{1}$-замкнутым (т.е. автодуальным прямым слагаемым в $\mathscr{N}$ ) и поэтому совпадает с $\mathscr{N}$ по предположению.

СЛЕДСТВИЕ 2. Пусть $\mathscr{M}$ автодуален $u\{\mathscr{N},\langle\cdot, \cdot\rangle\}$ произволен. Каждое инъективное модульное отображение из $\mathscr{M}$ в $\mathscr{N}$ является гильбертовим $A$-модульным изоморфизмом модуля $\mathscr{M}$ на прямое слагаемое в $\mathscr{N}$.

Для наших приложений в $\S 3$ нам понадобится следующий частный результат:

СЛЕДСТВИЕ 3. Пусть $\mathscr{M} u \mathscr{N}$ - счетно порожденнье А-модули, $a$ $F: \mathscr{M} \rightarrow \mathscr{N}$ - фредгольмов оператор (см. [13]). Тогда Ker $F u(\operatorname{Im} F)^{\perp}$ являются проективными конечно порожденными $A$-подмодулями, $a$ Ind $F=$ $[\operatorname{Ker} F]-\left[(\operatorname{Im} F)^{\perp}\right]$ в $K_{0}(A)$.

ДокаЗАтЕЛЬство. Пусть $\mathscr{\mathscr { M }}=\mathscr{M}_{0} \widehat{\oplus} \mathscr{M}_{1}, \mathscr{N}=\mathscr{N}_{0} \oplus \mathscr{N}_{1}-$ разложения из определения $A$-фредгольмова оператора:

$$
F=\left(\begin{array}{cc}
F_{0} & 0 \\
0 & F_{1}
\end{array}\right): \mathscr{M}_{0} \widehat{\oplus} \mathscr{M}_{1} \rightarrow \mathscr{N}_{0} \oplus \mathscr{N}_{1},
$$

$F_{0}: \mathscr{M}_{0} \cong \mathscr{N}_{0}, F_{1}: \mathscr{M}_{1} \rightarrow \mathscr{N}_{1}, \mathscr{M}_{1}$ и $\mathscr{N}_{1}$ являются проективными конечно порожденными модулями. Пусть $x=x_{0}+x_{1}, x_{0} \in \mathscr{M}_{0}$ и $x_{1} \in \mathscr{M}_{1}$, а $F(x)=0$, так что $0=F_{0}\left(x_{0}\right)+F_{1}\left(x_{1}\right) \in \mathscr{N}_{0} \oplus \mathscr{N}_{1}$. Таким образом, $F_{0}\left(x_{0}\right)=0, F_{1}\left(x_{1}\right)=0$, а значит, $x_{0}=0$ и $x \in \mathscr{M}_{1}$. Поэтому $\operatorname{Ker} F=\operatorname{Ker} F_{1} \subset \mathscr{M}_{1}$. По лемме $2 \operatorname{Ker} F$ является проективным конечно порожденным $A$-модулем и имеет ортогональное дополнение. Таким образом, по следствию 2

$$
F=\left(\begin{array}{ccc}
F_{0} & 0 & 0 \\
0 & F_{1}^{\prime} & 0 \\
0 & 0 & 0
\end{array}\right): \mathscr{M}_{0} \widehat{\oplus} \mathscr{M}_{1}^{\prime} \oplus \operatorname{Ker} F \rightarrow\left(\mathscr{N}_{0} \oplus \overline{F\left(\mathscr{M}_{1}^{\prime}\right)}\right) \widehat{\oplus}(\operatorname{Im} F)^{\perp}
$$

и $\operatorname{Ind} F=[\operatorname{Ker} F]-\left[(\operatorname{Im} F)^{\perp}\right]$. 
Следующий пример показывает, что ситуация может быть совершенно иной в случае общих гильбертовых $\mathrm{C}^{*}$-модулей и их инъективных отображений.

Пример 3. Рассмотрим $\mathrm{C}^{*}$-алгебру $A=C([0,1])$ всех непрерывных функций на отрезке $[0,1]$ как автодуальный гильбертов $A$-модуль над ней самой со стандартным $A$-значным скалярным произведением $\langle a, b\rangle_{A}=a b^{*}$. Отображение $\phi: f(x) \rightarrow x \cdot f(x)(x \in[0,1])$ является инъективным ограниченным модульным морфизмом. Его образ имеет тривиальное ортогональное дополнение, но он не замкнут по норме и, следовательно, не является прямым слагаемым в $A$. Его замыкание также не является прямым слагаемым в $A$. Тем не менее биортогональное дополнение образа отображения $\phi$ в $A$ совпадает с $A$.

Лемма 3. Пусть $\mathscr{P}$ и $\mathscr{Q}-$ автодуальнье гильбертовь $A$-подмодули модуля $\mathscr{M}$. Тогда $\mathscr{P} \cap \mathscr{Q}$ является автодуальным гильбертовым А-модулем и прямьлм слагаемьл в $\mathscr{M}$. Более того, $\mathscr{P}+\mathscr{Q} \subseteq \mathscr{M}-$ автодуальный гильбертов А-подмодуль.

Если Я является проективным и конечно порожденным, то пересечение $\mathscr{P} \cap \mathscr{Q}$ также проективно и конечно порождено. Если и $\mathscr{P}$, и $\mathscr{Q}$ проективны и конечно порождены, то теми же свойствами обладает и их сумма $\mathscr{P}+\mathscr{Q}$.

ДокаЗАТЕЛЬСТво. Пусть $p: \mathscr{M}=\mathscr{P} \oplus \mathscr{P}^{\perp} \rightarrow \mathscr{P}^{\perp}$ - каноническая ортогональная проекция, существующая по [3, Theorem 2.8] (cp. [2] в проективном случае). Пусть $p_{\mathscr{Q}}=p: \mathscr{Q} \rightarrow \mathscr{P}^{\perp}$. Поскольку $\mathscr{Q}-$ автодуальный гильбертов $A$-модуль, оператор $p_{\mathscr{Q}}$ допускает сопряженный и $\operatorname{Ker} p_{\mathscr{Q}} \subseteq \mathscr{Q}$ является прямым слагаемым по лемме 2. Следовательно, он представляет собой автодуальный гильбертов $A$-подмодуль $\mathscr{Q} \subseteq \mathscr{M}$. Но $\operatorname{Ker} p_{\mathscr{Q}}=\mathscr{P} \cap \mathscr{Q}$.

Для доказательства второго утверждения надо снова воспользоваться тем фактом, что всякий автодуальный гильбертов $A$-подмодуль является прямым слагаемым (ср. [3]).

Если $\mathscr{P}$ является проективным и конечно порожденным, то этими же свойствами будет обладать и каждое прямое слагаемое в нем. Это замечание дает оставшиеся утверждения.

\section{§3. Числа Лефшеца}

В этом параграфе мы считаем, что $A$ является $\mathrm{W}^{*}$-алгеброй. Это ограничение позволяет нам применить результаты предыдущего параграфа, которые, вообще говоря, верны только в $\mathrm{W}^{*}$-случае. Циклические гомологии мы считаем топологизированными при помощи проективной топологии в тензорном произведении.

Пусть $U$ - унитарный оператор в проективном конечно порожденном гильбертовом $A$-модуле $\mathscr{P}$. Тогда

$$
U=\int_{S^{1}} e^{i \varphi} d P(\varphi)
$$

где $P(\varphi)$ - проекторнозначная мера, принимающая значения в $\mathrm{W}^{*}$-алгебре всех ограниченных (допускающих сопряженный) модульных операторах в $\mathscr{P}$, а интеграл сходится по отношению к норме. Таким образом, мы имеем ограниченную 
и монотонную функцию

$$
L(\mathscr{P}, U): S^{1} \rightarrow K_{0}(A), \quad \varphi \mapsto[P(\varphi)]
$$

по отношению к частичному порядку в пространстве проекторов. Обозначим множество таких функций через $K_{0}(A)_{S}$.

Заметим, что можно считать числа Лефшеца для действия компактной группы, рассмотренные в [19], принимающими значения (для унитарного представления) в подпространстве конечнозначных (простых) функций

$$
\operatorname{Simlpe}\left(S^{1}, K_{0}(A)\right) \subset K_{0}(A)_{S} \text {. }
$$

Предположим, что $\mathscr{P}=A^{n}$. Мы ставим в соответствие интегральной сумме интеграла (1)

$$
\sum_{k} e^{i \varphi_{k}} P\left(\mathscr{E}_{k}\right)
$$

следующий класс циклических гомологий $H C_{2 l}(M(n, A))$ :

$$
\sum_{k} P\left(\mathscr{E}_{k}\right) \otimes \cdots \otimes P\left(\mathscr{E}_{k}\right) \cdot e^{i \varphi_{k}}
$$

Переходя к пределу, мы получаем такой элемент:

$$
\widetilde{T} U=\int_{S^{1}} e^{i \varphi} d(P \otimes \cdots \otimes P)(\varphi) \in H C_{2 l}(M(n, A)) .
$$

Положим

$$
T(U)=\operatorname{Tr}_{*}^{n}(\widetilde{T} U) \in H C_{2 l}(A),
$$

где $\operatorname{Tr}_{*}^{n}$ - след в циклических гомологиях.

ЛЕмма 4 [19, лемма 6.1]. Пусть $J: \mathscr{M}=A^{m} \rightarrow \mathscr{N}=A^{n}$ - изоморфизм, $U_{\mathscr{M}}: \mathscr{M} \rightarrow \mathscr{M}, U_{\mathscr{N}}: \mathscr{N} \rightarrow \mathscr{N}$ суть A-унитарные операторьи, причем $U_{\mathscr{M}}=$ $U_{\mathscr{N}} J$. Tогда

$$
T\left(U_{\mathscr{M}}\right)=T\left(U_{\mathscr{N}}\right)
$$

Сходная техника может быть развита и для проективного конечно порожденного $A$-модуля $\mathscr{N}$ вместо $A^{n}$. Для этого мы положим $\mathscr{N}=q\left(A^{n}\right)$, где $q$ обозначает ортогональную проекцию модуля $A^{n}$ на его прямое ортогональное слагаемое $\mathscr{N}$. Положим

$$
\begin{aligned}
U \oplus 1: A^{n} \cong \mathscr{N} \oplus(1-q) A^{n} \rightarrow \mathscr{N} \oplus(1-q) A^{n} \cong A^{n}, \\
\widetilde{T} U=\int_{S^{1}} e^{i \varphi} d(q P q \otimes \cdots \otimes q P q)(\varphi) .
\end{aligned}
$$

Корректность является немедленным следствием леммы 4.

Рассмотрим $A$-эллиптический комплекс $(E, d)$ и его унитарный эндоморфизм $U$. Результаты $\S 2$ (см. предложение 2 , следствие 3 , лемму 3 ) и стандартные рассуждения теории Ходжа дают нам возможность доказать следующую лемму. 
Лемма 5. Для A-фредгольмова оператора

$$
F=d+d^{*}: \Gamma\left(\mathscr{E}_{\mathrm{ev}}\right) \rightarrow \Gamma\left(\mathscr{E}_{\mathrm{od}}\right)
$$

имеют место соотношения

$$
\begin{aligned}
& \operatorname{Ker}\left(\left.F\right|_{\Gamma\left(\mathscr{E}_{\mathrm{ev}}\right)}\right) \stackrel{\text { def }}{=} H_{\mathrm{ev}}(\mathscr{E})=\oplus H_{2 i}(\mathscr{E}), \\
& \operatorname{Ker}\left(\left.F\right|_{\Gamma\left(\mathscr{E}_{\text {od }}\right)}\right) \stackrel{\text { def }}{=} H_{\mathrm{od}}(\mathscr{E})=\oplus H_{2 i+1}(\mathscr{E}),
\end{aligned}
$$

где $H_{m}(\mathscr{E})$ - ортогональное дополнение $\kappa \operatorname{Im} d \subset \operatorname{Ker} d \subset \Gamma\left(\mathscr{E}_{m}\right), u H_{m}(\mathscr{E})$ - проективные $U$-инвариантные гильбертовы $A$-модули.

ДоказАТЕльство. Для $u_{2 i} \in \Gamma\left(E_{2 i}\right)$ в силу соотношения

$$
\left(d+d^{*}\right)\left(u_{0}+u_{2}+u_{4}+\cdots\right)=0
$$

мы получаем

$$
d u_{0}+d^{*} u_{2}=0, \quad d u_{2}+d^{*} u_{4}=0, \quad \ldots .
$$

Вместе с равенством

$$
\left(d u, d^{*} v\right)=\left(d^{2} u, v\right)=0
$$

это дает

$$
d u_{0}=0, \quad d u_{2}=0, \quad \ldots, \quad d^{*} u_{2}=0, \quad d^{*} u_{4}=0, \quad \ldots,
$$

что влечет за собой соотношение $u_{2 i} \in \operatorname{Ker}\left(d+d^{*}\right)$. С другой стороны, для $v_{2} \in \operatorname{Im} d, v_{2}=d v_{1}$ выполняются равенства

$$
\left(v_{2}, u_{2}\right)=\left(d v_{1}, u_{2}\right)=\left(v_{1}, d^{*} u_{2}\right)=0 .
$$

Значит, $u_{2 i} \in H_{2 i}(E)$. Обратно, пусть $u=u_{0}+u_{2}+\ldots, u_{2 i} \in H_{2 i}(E)$, т. е. $d u_{2 i}=0(i=0,1,2, \ldots)$ и для любого $v_{2 i-1} \in E_{2 i-1}$

$$
\left(d v_{2 i-1}, u_{2 i}\right)=0, \quad\left(v_{2 i-1}, d^{*} u_{2 i}\right)=0,
$$

так что $d^{*} u_{2 i}=0$. Значит, $u \in \operatorname{Ker}\left(d+d^{*}\right)$. Инвариантность и проективность следуют из доказанного отождествления и следствия 3.

ОПРЕДЕЛЕНИЕ 1. МЫ определим иисло Лефшеца $L_{1}$ формулой

$$
L_{1}(\mathscr{E}, U)=\sum_{i}(-1)^{i} T\left(U \mid H_{i}(\mathscr{E})\right) \in K_{0}(A)_{S} .
$$

ОПРЕДЕЛЕНИЕ 2. Мы определим число Лефшеца $L_{2 l}$ формулой

$$
L_{2 l}(\mathscr{E}, U)=\sum_{i}(-1)^{i} T\left(U \mid H_{i}(\mathscr{E})\right) \in H C_{2 l}(A) .
$$

После всего сказанного следуюшая теорема очевидна.

Теорема 1. Пусть характер Чженя Сh определен, как в $[1,6,7]$. Тогда

$$
L_{2 l}(\mathscr{E}, U)=\int_{S^{1}}\left(\mathrm{Ch}_{2 l}^{0}\right)_{*}\left(L_{1}(\mathscr{E}, U)\right)(\varphi) d \varphi .
$$

ЗАмЕчАниЕ 1 . В ситуациях, когда эндоморфизм $V$ эллиптического $\mathrm{C}^{*}$-комплекса является элементом представленной на этом комплексе аменабельной группы $G, A$-значные скалярные произведения могут быть выбраны $G$-инвариантными, что дает унитарность $V$ (см. [11]). Однако имеется еще одно препятствие, требующее новых подходов, и оно будет описано в примере 4 ниже. 


\section{§4. Препятствия в $\mathrm{C}^{*}$-случае и близкие вопросы}

Цель настоящего параграфа - продемонстрировать некоторые препятствия, возникающие в теории общих гильбертовых $\mathrm{C}^{*}$-модулей для $\mathrm{C}^{*}$-алгебр $A$, более общих, чем $\mathrm{W}^{*}$-алгебры, которые вынудили нас налагать определенные ограничения в рассмотрениях предыдущего параграфа. Для работы в общем $\mathrm{C}^{*}$-случае часто бывает полезным следующая базовая конструкция, введенная В. Л. Пашке и Х. Лином. Она устанавливает связь между $\mathrm{W}^{*}$-случаем и общим $\mathrm{C}^{*}$-случаем.

ЗАмечание 2 (ср. [9, Definition $1.3 ; 14, \S 4]$ ). Пусть $\{\mathscr{M},\langle\cdot, \cdot\rangle\}$ - левый предгильбертов $A$-модуль над заданной $\mathrm{C}^{*}$-алгеброй $A$. Алгебраическое тензорное произведение $A^{* *} \otimes \mathscr{M}$ становится левым $A^{* *}$-модулем, если определить действие $A^{* *}$ на его элементарных тензорах по формуле $a b \otimes h=a(b \otimes h)$ для $a, b \in A^{* *}, h \in \mathscr{M}$. Теперь, полагая

$$
\left[\sum_{i} a_{i} \otimes h_{i}, \sum_{j} b_{j} \otimes g_{j}\right]=\sum_{i, j} a_{i}\left\langle h_{i}, g_{j}\right\rangle b_{j}
$$

на конечных суммах элементарных тензоров, получим вырожденное $A^{* *}$-значное скалярное предпроизведение. При факторизации модуля $A^{* *} \otimes \mathscr{M}$ по $N=$ $\left\{z \in A^{* *} \otimes \mathscr{M}:[z, z]=0\right\}$ получается предгильбертов $A^{* *}$-модуль, обозначаемый далее через $\mathscr{M}^{\#}$. Он содержит $\mathscr{M}$ в качестве $A$-подмодуля. Если $\mathscr{M}$ является гильбертовым, то и $\mathscr{M}^{\#}$ гильбертов, и наоборот. Перенос автодуальности сложнее. Если $\mathscr{M}$ автодуален, то и $\mathscr{M}^{\#}$ автодуален. Однако возникает

Вопрос 1. Предположим, что наша $\mathrm{C}^{*}$-алгебра $A$ унитальна. Следует ли из автодуальности модуля $\mathscr{M}^{\#}$, что исходный модуль $\mathscr{M}$ уже был автодуальным?

Другие стандартные свойства, такие, например, как $\mathrm{C}^{*}$-рефлексивность, не могут быть перенесены. Но каждый ограниченный $A$-линейный оператор $T$ в $\mathscr{M}$ имеет единственное продолжение до $A^{* *}$-линейного оператора в $\mathscr{M}^{\#}$, coхраняющее операторную норму (cp. [9, Definition 1.3]).

ПрЕДЛОЖЕНИЕ 3 . Пусть $\phi: \mathscr{M} \rightarrow \mathscr{N}$ - ограниченное $A$-линейное отображение. Тогда ядро $\operatorname{Ker}(\phi)$ морфизма ф совпадает с его биортогональным дополнением в $\mathscr{M}$. Вообще говоря, оно не является прямим слагаемьм.

ДокАЗАтЕльство. Предположим, что $\operatorname{Ker}(\phi) \neq \operatorname{Ker}(\phi)^{\perp \perp}$ по отношению к $A$-значному скалярному произведению в $\mathscr{M}$. Образуем прямую сумму $\mathscr{L}=$ $\mathscr{M} \oplus \mathscr{N}$. Отображение $\phi$ продолжается до ограниченного $A$-линейного отображения $\psi$ на $\mathscr{L}$, если положить

$$
\psi(x)= \begin{cases}\phi(x), & x \in \mathscr{M}, \\ 0, & x \in \mathscr{N} .\end{cases}
$$

Далее, продолжим $\psi$ до ограниченного $A^{* *}$-линейного оператора в соответствующем гильбертовом $A^{* *}$-модуле $\mathscr{L}^{\#}$. По лемме 2 оба множества $\operatorname{Ker}(\phi)^{\#}$ и $\left(\operatorname{Ker}(\phi)^{\perp \perp}\right)^{\#}$ содержатся в ядре $\operatorname{Ker}(\psi)$ отображения $\psi$, которое является прямым слагаемым в $\mathscr{L}^{\#}$ и удовлетворяет соотношению $\operatorname{Ker}(\psi)=\operatorname{Ker}(\psi)^{\perp \perp}$. Это противоречит предположению. Второе утверждение следует из примера 1.

СлЕДСТВИЕ 4. Ядро $\operatorname{Ker}(r)$ яюбого ограниченного модульного отображения $r: \mathscr{M} \rightarrow A$ совпадает с его биортогональньм дополнением в $\mathscr{M}$, но, вообще говоря, не является прямым слагаемьм. 
СЛЕДСТВИЕ 5. Предположим, что имеется ограниченное модульное отображение $r: \mathscr{M} \rightarrow A$ на $\mathscr{M}$ со свойством $\operatorname{Ker}(r)^{\perp}=\{0\}$. Тогда $r$ является нулевым.

Лемма 6. Пусть $\{\mathscr{M},\langle\cdot, \cdot\rangle\}$ является (левьлм) гильбертовылм $A$-модулем. Для каждого ограниченного модульного отображения $r: \mathscr{M} \rightarrow A$ подмножество $\operatorname{Ker}(r)^{\perp} \subseteq \mathscr{M}$ являетсл гильбертовым $A$-подмодулем, изомор $\phi$ ньи как аильбертов $A$-модуль замкнутому по норме (левому) идеалу $D$ в $A$, снабженному стандартным $A$-значнылм скалярным произведением $\langle\cdot, \cdot\rangle_{A}$.

ДоКАЗАТЕЛЬСТво. По следствию 3 множество $\operatorname{Ker}(r)^{\perp} \subseteq \mathscr{M}$ может без ограничения обшности считаться ненулевым. Снова образуем гильбертов $A^{* *}$-модуль $\mathscr{M}^{\#}$ и продолжим $r$ до ограниченного $A^{* *}$-линейного отображения $r^{\prime}$ на нем. Ядро отображения $r^{\prime}$ является прямым слагаемым в $\mathscr{M}^{\#}$, изоморфным (левому) замкнутому по норме идеалу $A^{* *}$ как гильбертов $A^{* *}$-модуль по следствию 1 и предложению 1. Следовательно, $\operatorname{Ker}(r) \subseteq \operatorname{Ker}\left(r^{\prime}\right) \cap \mathscr{M} \subseteq \mathscr{M}^{\#}$ изоморфно (левому) замкнутому по норме идеалу $D$ в $A$ как (левый) гильбертов $A$-модуль.

Мы хотели бы получить структурную теорему о взаимоотношении гильбертовых $\mathrm{C}^{*}$-модулей и их $\mathrm{C}^{*}$-сопряженных банаховых $\mathrm{C}^{*}$-модулей. Чтобы получить полную картину, введем следующую новую топологию на (левых) гильбертовых $\mathrm{C}^{*}$-модулях по аналогии с (правой) строгой топологией на $\mathrm{C}^{*}$-алгебре $A$.

ОПРЕДЕлЕНИЕ 3 . Пусть $\{\mathscr{M},\langle\cdot, \cdot\rangle\}$ является (левым) гильбертовым $A$ модулем. Ограниченная по норме сеть $\left\{x_{\alpha}: \alpha \in I\right\}$ элементов из $\mathscr{M}$ называется фундаментальной по отношению $к$ правой *-строгой топологии, если сеть $\left\{\left\langle y, x_{\alpha}\right\rangle: \alpha \in I\right\}$ является сетью Коши по отношению к топологии нормы на $A$ для любого $y \in \mathscr{M}$. Сеть $\left\{x_{\alpha}: \alpha \in I\right\}$ сходится $\kappa$ әлементу $x \in \mathscr{M}$ в правой *-строгой топологии тогда и только тогда, когда

$$
\lim _{\alpha \in I}\left\|\left\langle y, x-x_{\alpha}\right\rangle\right\|_{A}=0
$$

для любого $y \in \mathscr{M}$.

Теорема 2. Пусть $\{\mathscr{M},\langle\cdot, \cdot\rangle\}$ является (левьлм) гильбертовылм $A$-модулем. Следуюшие условия әквивалентны:

(1) $\mathscr{M}$ автодуален;

(2) единичный шар модуля $\mathscr{M}$ полон в правой *-строгой топологии. Кроме того, линейная оболочка пополненного по правой *-строгой топологии единичного шара модуля $\mathscr{M}$ совпадает с $A$-сопряженным $\kappa \mathscr{M}$ банаховъцм $A$-модулем $\mathscr{M}^{\prime}$.

ДокАЗАТЕЛЬСтво. Предположим, что единичный шар модуля $\mathscr{M}$ полон в правой $*$-строгой топологии. Рассмотрим произвольное нетривиальное ограниченное модульное отображение $r: \mathscr{M} \rightarrow A$ с нормой, равной единице. Сосредоточим внимание на ненулевом гильбертовом $A$-подмодуле $\operatorname{Ker}(r)^{\perp} \subseteq \mathscr{M}$, который по лемме 6 изоморфен как гильбертов $A$-модуль замкнутому по норме (левому) идеалу $D$ в $A$, снабженному стандартным $A$-значным скалярным произведением $\langle\cdot, \cdot\rangle_{A}$. По [3, Theorem 3.2] имеется сеть $\left\{x_{\alpha}: \alpha \in I\right\} \subset \operatorname{Ker}(r)^{\perp}$, ограниченная единицей по норме и такая, что $\tau_{2}-\lim _{\alpha \in I} x_{\alpha}=r$ в автодуальном гильбертовом $A^{* *}$-модуле $\left(\left(\operatorname{Ker}(r)^{\perp}\right)^{\#}\right)^{\prime}$. Но все значения $r(y), y \in \operatorname{Ker}(r)^{\perp}$, принадлежат $A$ и, в частности, множеству всех правых мультипликаторов $\mathrm{C}^{*}$-подалгебры и двустороннего идеала $B=\left\langle\operatorname{Ker}(r)^{\perp}, \operatorname{Ker}(r)^{\perp}\right\rangle$ в $A$. Следовательно, 
существует такая сеть $\left\{x_{\alpha}: \alpha \in I\right\} \subset \operatorname{Ker}(r)^{\perp}$, что предел по $\|\cdot\| \mathscr{M}$

$$
\lim _{\alpha \in I} b\left(\left\langle y, x_{\alpha}\right\rangle-r(y)\right)=0
$$

для каждого $y \in \mathscr{M}$ и каждого $b \in B($ ср. $[16, \S 3.12])$, где условие $y \in \mathscr{M} \subset \mathscr{M}^{\#}$ может подразумеваться в силу следствия 1. Поскольку множество $\{b y: b \in B$, $\left.y \in \operatorname{Ker}(r)^{\perp}\right\}$ плотно по норме в $\operatorname{Ker}(r)^{\perp}$, одна импликация доказана. Противоположная следует из формулы для предела по $\|\cdot\|_{A}$

$$
r(y)=\lim _{\alpha \in I}\left\langle y, x_{\alpha}\right\rangle, \quad y \in \mathscr{M},
$$

для $y \in \mathscr{M}$, определяющей ограниченное модульное отображение $r: \mathscr{M} \rightarrow A$ для каждой ограниченной по норме фундаментальной по отношению к правой *-строгой топологии сети $\left\{x_{\alpha}: \alpha \in I\right\} \subset \mathscr{M}$. Попутно заключаем, что $A$-сопряженный банахов $A$-модуль $\mathscr{M}^{\prime}$ для всякого гильбертова $A$-модуля $\mathscr{M}$ совпадает с линейной оболочкой единичного шара в $\mathscr{M}$, пополненного по правой $*$-строгой топологии.

СЛЕДСТВИЕ 6. Пусть $D-$-замкнутый по норме (левый) идеал в А. Тогда $\left\{D,\langle\cdot, \cdot\rangle_{A}\right\}$ является автодуальным в том и только том случае, когда суиествует такой проектор $p \in A$, ито $D \equiv A p$ u $p \in D$.

ДокАЗАтЕльство. Если $D$ автодуален, то тождественное вложение $D$ в $A$ является ограниченным $A$-линейным отображением, допускающим сопряженный. Он должен представляться элементом $p \in D$ со свойством $d p^{*}=d$ для каждого $d \in D$. Таким образом, $p p^{*}=p \in D$ - положительный идемпотент. Линейные свойства отображения $p$ определяют структуру $D$ как $D \equiv A p$.

Теорема 3. Пусть $\{\mathscr{M},\langle\cdot, \cdot\rangle\}-(л е в ь и ̆) ~ а и л ь б е р т о в ~ А-м о д у л ь . ~ Е с л и ~ \mathscr{M ~}$ является А-рефлексивньм, то каждая ограниченная по норме сеть $\left\{x_{\alpha}: \alpha \in I\right\}$ элементов из $\mathscr{M}$, для которой все сети вида $\left\{r\left(x_{\alpha}\right): \alpha \in I\right\}$ $\left(r \in \mathscr{M}^{\prime}\right)$ сходятся по $\|\cdot\|_{A}$, имеет предел $x$, реализуюший $r(x)=\lim _{\alpha \in I} r\left(x_{\alpha}\right)$ в $\mathscr{M}$. Обратное, вообше говоря, неверно.

ДокАЗАТЕЛьСтво. Будем считать, что $\mathscr{M}$ не является автодуальным, поскольку в противном случае мы можем просто воспользоваться теоремой 2. Очевидно, что линейная оболочка замыкания единичного шара модуля $\mathscr{M}$ по отношению к указанной топологии является банаховым $A$-модулем $\mathscr{N}$. Продолжим $A$-значное скалярное произведение с $\mathscr{M}$ на $\mathscr{N}$ по правилу

$$
\langle x, y\rangle=\lim _{\alpha \in I}\left\langle x_{\alpha}, y\right\rangle
$$

для каждого элемента $\langle\cdot, y\rangle \in \mathscr{M}^{\prime}$, где $y \in \mathscr{M}$. Поскольку сеть сходится также по отношению к правой *-строгой топологии на $\mathscr{M}$, предел $x$ может рассматриваться как $A$-линейный ограниченный функционал на $\mathscr{M}$. Это позволяет определить значение $\langle x, x\rangle$ тем же способом. Следовательно, $\mathscr{N}$ является гильбертовым $A$-модулем, содержащим $\mathscr{M}$ как гильбертов $A$-подмодуль и имеющим тот же самый $A$-сопряженный банахов $A$-модуль $\mathscr{M}^{\prime} \equiv \mathscr{N}^{r}$ (cp. [15], где имеются схожие конструкции). Кроме того, единичный шар модуля $\mathscr{N}$ полон в новой топологии. Поскольку $A$-значное скалярное произведение на $\mathscr{M}$ может быть 
продолжено до $A$-значного скалярного произведения на $\mathscr{M}^{\prime \prime} \equiv \mathscr{N}^{\prime \prime}$ по $[15$, Theorem 2.4], то каждый элемент из $\mathscr{M}^{\prime \prime}$ может быть описан таким способом и $\mathscr{N}$ является $A$-рефлексивным.

Пусть $A$ есть $\mathrm{C}^{*}$-алгебра всех ограниченных линейных операторов в сепарабельном гильбертовом пространстве и $\mathscr{M}$ - множество всех компактных операторов в нем. Пусть $A$ действует на $\mathscr{M}$ умножением слева и $\mathscr{M}$ снабжено стандартным $A$-значным скалярным произведением, наследуемым с $A$. Тогда $\mathscr{M}$ превращается в левый гильбертов $A$-модуль. Очевидно, что $\mathscr{M}^{\prime}=\mathscr{M}^{\prime \prime}=A$. Но единичный шар модуля $\mathscr{M}$ замкнут по отношению к описанной выше топологии, поскольку, например, единица $\mathrm{C}^{*}$-алгебры $A$ не является компактным оператором и не может быть реализована описанным выше пределом.

ПримеР 4. Рассмотрим $\mathrm{C}^{*}$-алгебру $A=C([0,1])$ всех непрерывных функций на единичном отрезке как гильбертов $A$-модуль над собой. Пусть унитарный оператор $U$ определен формулой

$$
U(f)(t)=e^{i t} f(t), \quad t \in[0,1] .
$$

Примем этот унитарный оператор за образующую унитарного представления аменабельной (абелевой) группы $\mathbb{Z}$. Все комплексные неприводимые представления группы $\mathbb{Z}$ одномерны. Если бы мы захотели применить теорему А. С. Мишенко [12] в этом случае, то нам бы понадобилось наличие конечного спектра у порождающего элемента представления $U$, что не так в данном случае. Кроме того, единственными проекторами в $A$ и, следовательно, единственными самосопряженными идемпотентными модульными операторами на $A$ являются $1_{A}$ и $0_{A}$ и не существует спектрального разложения элементов и нет нетривиальных прямых $A$-модульных слагаемых в $A$.

ЗАмечание 3 . Как известно, во всех важных случаях морфизм $S$ дает изоморфизм между $H C_{2 l}(A)$ и $H C_{0}(A)$, так что мы можем работать только со второй группой. В этой ситуации мы можем определить число Лефшеца $L_{0} \in$ $H C_{0}(A)$ способом из [18] для общей $\mathrm{C}^{*}$-алгебры $A$.

Но для $K$-значных чисел даже в случае действия, например, аменабельной группы $G$ (см. пример 4), нам нужна бесконечность и сходимость в каком-то смысле, так что мы должны переходить к $K_{0}(A)_{S}$. Естественным выражением этой бесконечности собственных значений является спектральное разложение, так что мы должны работать с $\mathrm{W}^{*}$-алгебрами, по крайней мере для $L_{1}$. Решающим моментом является отсутствие в такой ситуации теоремы типа [12].

Конечно, это совершенно не строгие рассуждения, и мы имеем некоторую надежду на улучшение результатов, например, для монотонно полных $\mathrm{C}^{*}$-алгебр. Однако техника для монотонно полного случая довольно сложна, а результаты ненамного отличаются от полученных в $\mathrm{W}^{*}$-случае (cp. [4]).

\section{ЛитеРАтУРА}

1. Connes A. Non-commutative differential geometry. Publ. Math. IHES, 62, 41-144 (1985).

2. Dupré M. J., Fillmore P. A. Triviality theorems for Hilbert modules. In: Topics in modern operator theory, 5th International conference on operator theory. Timisoara 
and Herculane (Romania), June 2-12, 1980, Birkhäuser, Basel-Boston-Stuttgart, 1981, pp. 71-79.

3. Frank $M$. Self-duality and $\mathrm{C}^{*}$-reflexivity of Hilbert $\mathrm{C}^{*}$-modules. Z. Anal. Anwendungen, 9, 165-176 (1990).

4. Frank $M$. Hilbert $\mathrm{C}^{*}$-modules over monotone complete $\mathrm{C}^{*}$-algebras. Math. Nachr., 175, 61-83 (1995).

5. Havet J.-F. Calcul fonctionnel continu dans les modules hilbertiens autoduaux. Preprint, Université d'Orléans, Orléans, France, 1988.

6. Karoubi $M$. Homologie cyclique des groupes et des algébres. C. R. Acad. Sci. Paris Sér. 1, 297, 381-384 (1983).

7. Karoubi $M$. Homologie cyclique et $K$-théorie algébrique. I. C. R. Acad. Sci. Paris Sér. 1, 297, No. 8, 447-450 (1983).

8. Lance E. C. Hilbert $\mathrm{C}^{*}-$ Modules-a Toolkit for Operator Algebraists. Lect. Notes, University of Leeds, School of Mathematics, Leeds, England, 1993.

9. Lin $H$. Bounded module maps and pure completely positive maps. J. Operator Theory, 26, 121-138 (1991).

10. Lin H. Injective Hilbert $C^{*}$-modules. Pacif. J. Math., 154, 131-164 (1992).

11. Manuilov V. M. Representability of Functionals and Adjointability of Operators on $C^{*}$-Hilbert Modules. Preprint 1/94, Moscow State University, Dept. Mech. and Math., Seminar "Toplology and Analysis", Moscow, Russia, Sept. 1994.

12. Мищенко A. C. Представления компактных групп в гильбертовых модулях над $\mathrm{C}^{*}$-алгебрами. Труды Матем. ин-та им. В. А. Стеклова, 166, 161-176 (1984).

13. Мищенко A. C., Фоменко A. T. Индекс эллиптических операторов над C*-алгебрами. Изв. АН СССР, сер. матем., 43, №4, 831-859 (1979).

14. Paschke W. L. Inner product modules over $\mathrm{B}^{*}$-algebras. Trans. Am. Math. Soc., 182, 443-468 (1973).

15. Paschke $W$. L. The double $B$-dual of an inner product module over a $\mathrm{C}^{*}$-algebra $B$. Can. J. Math., 26, 1272-1280 (1974).

16. Pedersen G. K. C*-Algebras and their Automorphism Groups, Academic Press, London-New York-San Francisco, 1979.

17. Troitsky E. V. The index of equivariant elliptic operators over $\mathrm{C}^{*}$-algebras. Ann. Global Anal. Geom., 5, No. 1, 3-22 (1987).

18. Troitsky E. V. Lefschetz numbers of $\mathrm{C}^{*}$-complexes. In: Lect. Notes in Math., Vol. 1474, Springer, 1991, pp. 193-206.

19. Troitsky E. V. Orthogonal complements and endomorphisms of Hilbert modules and $\mathrm{C}^{*}$-elliptic complexes. In: Novikov Conjectures, Index Theorems and Ridgidity, Vol. 2 (London Math. Soc. Lect. Notes Series, Vol. 227), 1995, pp. 309-331.

Лейпцигский университет

Отдел матем. и информатики

Поступило в редакцию

e-mail: frank@mathematik.uni-leipzig.d400.de

31 мая 1995 г.

Московский государственный университет,

механико-математический факультет,

кафедра высшей геометрии и топологии

e-mail: troitsky@mech.math.msu.su 